\title{
Breastfeeding is associated with the risk of ovarian cancer in the UK Women's Cohort Study
}

\author{
F.N. Mohammed Danial ${ }^{1,3}$, J.E. Cade ${ }^{1}$, D.C. Greenwood ${ }^{2}$ and V.J. Burley ${ }^{1}$ \\ ${ }^{1}$ Nutritional Epidemiology Group, School of Food Science and Nutrition, University of Leeds, LS2 9JT United \\ Kingdom, ${ }^{2}$ Centre for Epidemiology and Biostatistics, University of Leeds, LS2 9JT United Kingdom and ${ }^{3}$ Faculty \\ of Hospitality, Tourism and Wellness, Universiti Malaysia Kelantan, Malaysia.
}

Studies have found that longer breastfeeding duration is associated with the risk of having ovarian cancer and other hormonal related cancers $^{(1)}$. However, the evidence is still inconsistent with a limited number of prospective studies providing evidence ${ }^{(2)}$. Therefore, this analysis aimed to explore the association between breastfeeding duration and the risks of ovarian cancer in a large prospective cohort of middle-aged women, the UK Women's Cohort Study (UKWCS).

The UKWCS participants were recruited in 1995-1998 at the age of 35-69 years ${ }^{(3)}$. Cancer incidence was obtained from the Office of National Statistics (ONS). A baseline questionnaire was used to estimate breastfeeding duration, ranging from $>0$ months to $>6$ months. Patients with prevalent cancers were excluded. The relationship between breast feeding duration and incidence of ovarian cancer was tested using survival analysis adjusting for age, children, socio-economic class, menopausal status, smoking status, body mass index, energy intake and age at menarche.

The total number of incident ovarian cancer cases was 224 over 16 years follow up. Mean age at recruitment was 54 years. Breastfeeding duration for each child shows that ever breastfeeding was associated with $6 \%$ risk reduction of ovarian cancer, after adjustment for confounders (HR: 0.94; $95 \%$ CI: 0.90 to 0.99 ). Further analysis on breastfeeding duration per child shows that the risk reduces by $43 \%$ among those who breastfed for more than 6 months per child (HR: $0 \cdot 57 ; 95 \%$ CI: $0 \cdot 35$ to $0 \cdot 95$ ). Additional analysis was done on cumulative breastfeeding duration across all children, with ovarian cancer risk, which shows a $2 \%$ risk reduction for every 1 month of breastfeeding (HR: 0.98; $95 \%$ CI: 0.97 to 1.00).

In conclusion, ever breastfeeding per child was associated with $6 \%$ risk reduction of ovarian cancer in a fully adjusted model and those who were breastfeeding for more than 6-month benefit more with a high-risk reduction of ovarian cancer by $43 \%$. This finding supports the World Health Organisation (WHO) recommendation for women to breastfeed for at least 6 months for each child. Future studies are warranted to determine if the duration of breastfeeding per child provides more meaningful data than cumulative breastfeeding duration. The average duration of breastfeeding per child might have more public health applicability than total duration and is a better reflection of lactation amenorrhea ${ }^{(1)}$.

1. Luan NN, Wu QJ, Gong TT et al. (2013) Breastfeeding and ovarian cancer risk: a meta-analysis of epidemiologic studies. Am J Clin Nutr 98, 1020-31. 2. Feng LP, Chen HL \& Shen MY (2014) Breastfeeding and the risk of ovarian cancer: a meta-analysis. J Midwifery Womens Health 59, 428-37. 3. Cade JE, Burley VJ, Alwan NA et al. (2017) Cohort Profile: The UK Women's Cohort Study (UKWCS). Int J Epidemiol 46, e11. 